Ciclos biogeoquímicos y fertilidad del suelo

\title{
Abundancia y actividad microbiana del suelo en sistemas silvopastoriles de la región chaqueña
}

Anriquez Analia ${ }^{1}$; Barreto Gabriela ${ }^{1}$; Silberman Juan ${ }^{1}$; Dominguez Nelson ${ }^{1}$; Dominguez Nuñez José ${ }^{2}$; Albanesi Ada ${ }^{1}$

${ }^{1}$ Facultad de Agronomía y Agroind - Univ. Nac. de Sgo. del Estero; ${ }^{2}$ ETSI Montes Univ. Politécnica de Madrid. ananriquez@hotmail.com

Introducción: La implementación de sistemas silvopastoriles (SP), generados por rolados de baja intensidad (RBI), en bosques secundarios de la región chaqueña, constituyen una alternativa sustentable para aumentar la productividad forrajera. El objetivo del trabajo fue evaluar la abundancia y actividad microbiana del suelo en SP de un año (SP1) y nueve años (SP9) de implantados, mediante RBI, y siembra simultánea de Panicum máximum cv Gatton panic, en dos sitios ecológicos de la región Chaqueña (Chaco semiárido y Chaco subhúmedo), en las estaciones húmeda $(\mathrm{H})$ y seca $(\mathrm{S})$ y bajo coberturas arbóreas de (Aspidosperma quebracho blanco (Qb), Schinopsis lorentzii (Qc) y Ziziphus mistol (M)).

Materiales y métodos: Se muestreó suelo en sistemas silvopastoriles de uno y nueve años y se utilizó como testigo bosque secundario $(\mathrm{T})$. Las variables de suelo evaluadas fueron, carbono de la biomasa microbiana (Cbm), actividades enzimáticas ureasa (Ureasa), hidrólisis de fluoresceína (FDA) y deshidrogenasa (Dh-asa) y la relación C:N. En el sitio ecológico del chaco semiárido el estudio se realizó bajo un experimento factorial $3 \times 3 \times 2$ en diseño completamente aleatorizado. Se estudiaron tres factores: usos del suelo (T, SP1 y SP9), coberturas (Qc, Qb y M) y estacionalidad (Hum y Seca), con tres repeticiones mientras que en el sitio ecológico del chaco subhúmedo el diseño fue $3 \times 2$, donde se estudiaron dos factores: usos del suelo (T, SP1 y SP9), y estacionalidad (H y S). Todas las variables fueron analizadas mediante ANOVA cuyas fuentes de variación fueron usos del suelo (T, SP1 y SP9), cobertura (Qc, Qb y M) (sólo para el Chaco semiárido) y estacionalidad (H y S). Se utilizó test de Duncan para la comparación de medias $(\alpha=0,05)$. Paquete estadístico InfoStat versión 2008, actualización 2011.

Resultados: La abundancia y actividad de los microorganismos del suelo se modifican, luego de un año de aplicado el RBI y siembra de $P$. máximum, sin embargo los mismos tienden a restaurarse a los nueve años. En el chaco semiárido en el primer año, en la estación húmeda, se produjo un aumento en el Cbm (40\%), en la actividad ureasa (70 \%), y se mantuvo constante la actividad FDA. Mientras que en el chaco subhúmedo disminuyó el Cbm (30\%) y la actividad FDA (40\%) y se mantuvo constante la actividad ureasa. Independientemente del sitio y de la estación, la actividad deshidrogenasa disminuyó en SP1 (aproximadamente un $30 \%$ ) como consecuencia del ingreso de residuos con alta relación C:N que provoca un estrés a los microorganismos. Debajo la cobertura de Mistol, se presentan mayores contenidos de Cbm y actividades enzimáticas.

Conclusiones: La actividad deshidrogenasa es independiente del sitio, contrariamente, la dirección de los cambios en las actividades ureasa y FDA son sitio dependientes y están influenciadas marcadamente por los pulsos de humedad, por el $\mathrm{C}$ orgánico total y por la relación $\mathrm{C}: \mathrm{N}$ de los residuos vegetales incorporados al suelo. De este estudio se deduce que la actividad deshidrogenasa sería la variable más sensible al cambio de uso del suelo. El suelo bajo la cobertura de mistol presenta mejor calidad en términos de abundancia y actividad microbiana. 